\title{
The Construction Contract Execution Through the Integration of Blockchain Technology
}

\author{
Giuseppe Martino Di Giuda, Giulia Pattini, Elena Seghezzi, \\ Marco Schievano and Francesco Paleari
}

\begin{abstract}
This paper aims to analyze the Blockchain level of implementation, focusing on the AEC sector that has always suffered from lack of trust, incomplete sharing, and transparency of information flow throughout the process execution. In this context, the progressive introduction of BIM based on the Blockchain technology can provide a trustworthy infrastructure for information management during the design, tender, and construction phases.
\end{abstract}

Keywords Blockchain $\cdot$ Smart contracts $\cdot$ Public tender $\cdot$ BIM $\cdot$ Information trust $\cdot$ Information immutability

\section{Introduction}

Blockchain can currently be considered as the main technology characterizing the digital transition observed within the most advanced world economies. Blockchain is a methodology capable of managing contracts and transactions through which assets are organized and guarded, social actions are governed and relations between nations, institutions, and individuals are guided (Shen and Pena-Mora 2018). Thanks to its nature, many public administrations are taking interest in the technology implementation, supporting Blockchain initiatives, setting goals and pursuing approaches that keep up with the technology (Carson et al. 2018; Kshetri 2018). Although various sectors are now exploring and testing Blockchain applications in their processes, its investigation in the construction sector is still meager and at a conceptual level (Mason 2017).

In an expanding construction sector, the maintenance of trust among stakeholders is difficult, the links are too complex hindering the information sharing with consequent waste of time and process costs (Institution of Civil Engineers, Blockchain

G. M. Di Giuda $(\varangle) \cdot$ G. Pattini $\cdot$ E. Seghezzi $\cdot$ M. Schievano · F. Paleari

Architecture, Built Environment and Construction Engineering-ABC Department, Politecnico di Milano, Milan, Italy

e-mail: giuseppe.digiuda@polimi.it

B. Daniotti et al. (eds.), Digital Transformation of the Design, Construction and Management Processes of the Built Environment, Research for Development, https://doi.org/10.1007/978-3-030-33570-0_3 
technology in the construction industry 2018); traditional information-sharing methods can influence negatively trust among participants, supply chain control, and asset management. In this context, the actual digital transition modifies and evolves the communication methods between project contractors. The actual BIM approach to the project guarantees the digital information exchange, offering a single database containing all the data created and shared by the operators during all phases of the construction process (Hsiao 2016). Building Information Modeling is in fact much more than an asset digital model because it defines the modalities of the model integration in the general construction system and the procedures through which information is added or extracted from the same and the creation, use, and management criteria of the informative model (Di Giuda and Villa 2016).

The progressive implementation of BIM models has fostered the project information exchange and collaboration among parties; however, the reliability and the transparency of each transition are not always guaranteed (Turk and Klinc 2017). Issues such as model property, right of modification and error responsibility, make it legitimate to assume an integration between BIM and Blockchain in order to overcome those limitations. In this dynamic context, Blockchain stands as a solution ensuring a transparent and precise information distribution among the participants by diverting the control of information by a single subject.

\section{Blockchain Technology}

Blockchain as a Distributed Ledger Technology (DLT) is a distributed data logging and maintenance system, that depends on and is ensured by the consensus mechanism implemented by the agents. The autonomy and updating of the information contained in the blocks are in fact subject to verification and authorization by all participants (Garzik and Donnelly 2018).

Consisting of a chain of blocks that develops within a distributed database, Blockchain prevents the structural alteration and the content violation, because the same information entered and validated in the chain are distributed, then stored in all nodes that compose it. Blockchain can be defined as a system that allows the data acquisition in a computer format, making it true and unchangeable thanks to the verification, validation, and control process carried out by the entire network through the consensus mechanism, and not by a third party (Kshetri 2017). The recent fast spread of the technology is mainly due to the benefits offered by its use, such as

- Intermediaries elimination;

- Information inalterability;

- Information traceability (Fig. 1). 


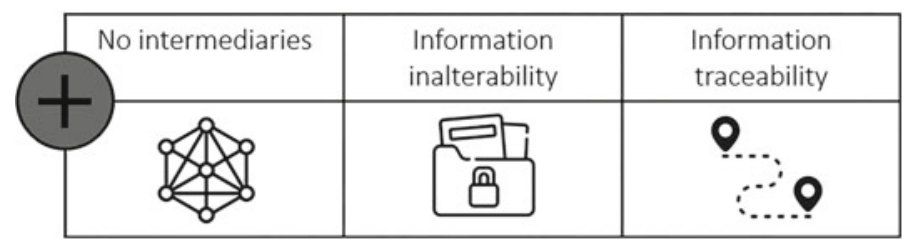

Fig. 1 Blockchain opportunities

\begin{tabular}{|l|l|l|}
\hline $\begin{array}{c}\text { Innovative } \\
\text { technology }\end{array}$ & Digitalized asset & $\begin{array}{l}\text { Operators } \\
\text { education }\end{array}$ \\
\hline & & \\
\hline
\end{tabular}

Fig. 2 Blockchain limits

Despite the advantages that Blockchain use offers, its youth creates challenges and obstacles that involve different areas and that can be identified with

- Innovative technology;

- Digitalized asset;

- Operators education (Fig. 2).

While Blockchain could solve some of the issues that arise with the use of BIM, one of its protocols has the potential to revolutionize the relationship between construction projects and to establish longstanding contractual procedures. Thanks to its programmable nature, the Blockchain allows the proper use of Smart Contract, i.e., contracts written in code capable of executing the clauses established and shared by the contracting parties automatically and independently. The implementation of a Smart Contract promotes the representation of clauses in the form of structured data executable by means of computer protocols with a high degree of accuracy compared to those defined by traditional language (Giancaspro 2017) (Fig. 3).

\begin{tabular}{|c|c|c|}
\hline \multicolumn{3}{|c|}{ Smart Contract } \\
\hline $\begin{array}{l}\text { Multiparty } \\
\text { agreement }\end{array}$ & Automatic codes & Immutable codes \\
\hline & 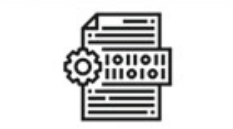 & 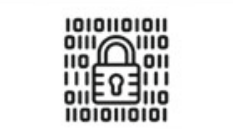 \\
\hline
\end{tabular}

Fig. 3 Smart contract based on Blockchain main features 


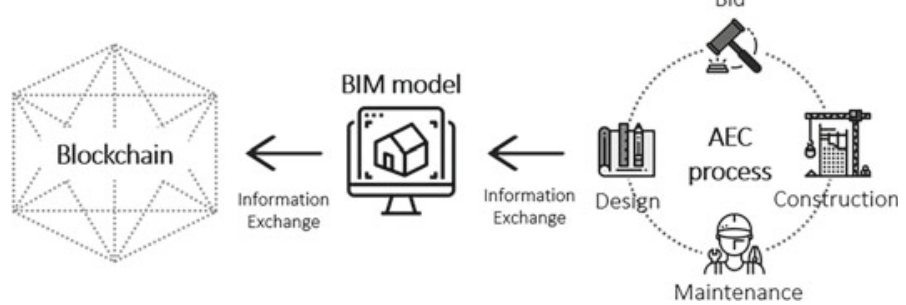

Fig. 4 BIM and Blockchain coordination

\section{Integration Between BIM and Blockchain}

Considering BIM methodology as a shared database among the project participants aimed at exchanging information on each process phase, it is fair to assume the activities of sharing, managing, and recording data by means of an information model supported by the Blockchain (Turk and Klinc 2017). The sharing information digital register offered by the technology ensures data reliability, integrity, and transparency by fostering loyal cooperation and trust among operators.

Since both the BIM approach and the Blockchain technology are based on the creation and management of a single source of information related to the process, it is legitimate to assume and investigate how to integrate them during the execution of the construction process. Due to the comparison and coordination of multiple disciplines, construction projects are often characterized by a large amount of data that can be stored in the BIM model, and ensured from the point of view of reliability and transparency if stored in the shared register offered by Blockchain. The combination of the information model and the distributed digital database makes it possible to create a single effective shared source of information relating to the project. This source can, therefore, be considered as the only source of truth that guarantees the data reliability, the congruence of the sources of information and the identity of the subjects responsible for the activities.

In this innovative context, the project BIM model is the only source of information, accessible and consultable by all participants, composed of reliable and unchangeable data. Since any information stored in the Blockchain database is traceable and unchangeable, time wasted and redundant verification of shared data due to frequent lack of trust among project participants are eliminated (Fig. 4).

\subsection{Benefits in the Construction Process}

The potential offered by the use of Blockchain allows removing the limitations that have recently discouraged the creation of the BIM model, such as reliability, traceability, disintermediation, recording of changes and data ownership. It is, therefore, 


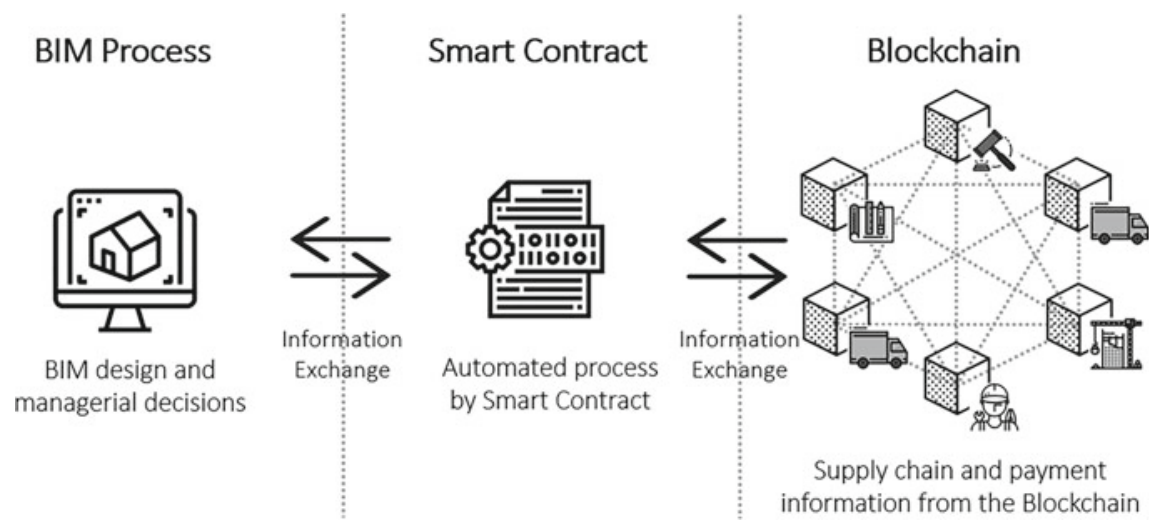

Fig. 5 BIM enables Blockchain technology

possible to highlight three main benefits that can be obtained through the joint use of BIM and Blockchain. First, the coordination between the BIM model and the distributed database containing all the process information ensures the creation of a single and reliable register, establishes a collaborative environment among all participants and defines transparently the responsibilities and duties of each, reducing or eliminating the emergence of any misunderstandings and subsequent conflicts between the parties. Second, to support the creation of a collaborative environment, the distributed database allows to store and trace the information intellectual property contained in it and entered by each party of the process. Third and last, if the two benefits illustrated above are mainly attributable to the design phase, the implementation of Smart Contract associated with the evolution of the BIM model is also relevant during the construction phase. The simultaneous progress between the BIM model and the execution of a Smart Contract makes it possible to automate all the delivery phases (Fig. 5).

The relationship among the parties involved in the whole process is often characterized by the presence of asymmetrical information during the design, tender, construction, and management phase, generating conflicts and mistrust with consequent impediments to the contract execution. For these reasons, the integration between the BIM model and the Blockchain illustrated so far is useful in making the activities carried out during the process explicit and visible, highlighting the honesty of those who act. Considering the benefits, various public administrations, including the Italian one, are committed to understanding the potential uses of the Blockchain in order to eliminate and manage in an optimal way the traditional criticalities. 


\subsection{Implementation in Contract Execution}

The BIM model development based on a distributed digital register, updated and modified during contract execution, allows us to archive all the transitions made by drawing up a non-modifiable chronology of all the construction process stages. The Blockchain properties, guaranteeing the unchangeability of the data and the presence of a widespread control of every single step of the procedure, are, therefore, well disposed to face the waste of time and cost due to the lack of trust and the absence of a transparent sharing of information between the participants in the different process stages.

The possible uses and therefore the advantages that can be obtained through the BIM model development to support all the contract execution phases-design, commissioning, construction, and management of the asset—based on the distributed database Blockchain are highlighted in Fig. 6.

\subsubsection{Design}

The design levels preparation based on BIM methods allows us to share and exchange all the project information through a single digital platform. The integration of these digital methods with the shared Blockchain database allows to create a truly collaborative process, that limits the gap between digital information modeling and the management of project information — administrative, financial, insurance - typically expressed in sheets. The possibility of associating automatic payments to the data in the model through the use of Smart Contract guarantees an exhaustive implementation of the engineering and architecture service contracts and therefore the respect of the requests expressed by each person involved. In this regard, the usual BIM model is no longer considered as a central data-sharing environment based on a cloud platform governed by a third party, but rather as a peer-to-peer data exchange environment, in which each participant can define and control the ownership of information in an immutable way.

\begin{tabular}{|l|l|l|l|}
\hline \multicolumn{1}{|c|}{ Design phase } & \multicolumn{1}{|c|}{ Bid phase } & \multicolumn{1}{c|}{ Construction phase } & \multicolumn{1}{c|}{ Life cycle phase } \\
\hline $\begin{array}{l}\text { Sharing and exchanging } \\
\text { project information in a } \\
\text { collaborative } \\
\text { environment }\end{array}$ & $\begin{array}{l}\text { Immutable and } \\
\text { transparent tender } \\
\text { documents }\end{array}$ & $\begin{array}{l}\text { Updating and } \\
\text { monitoring supply } \\
\text { chain and construction } \\
\text { progress }\end{array}$ & $\begin{array}{l}\text { Monitoring } \\
\text { maintenance and } \\
\text { circular economy } \\
\text { principles satisfaction }\end{array}$ \\
\hline
\end{tabular}

Fig. 6 BIM and Blockchain in contract execution phases 


\subsubsection{Bid}

Due to the frequent ambiguities that arise during tender procedures, the presence of a distributed ledger in which all information is stored in a transparent, permanent, and accessible manner helps to contain any misunderstanding. The digital tools implementation provided by the distributed platform, typical of the Blockchain technology, would make it possible to store in an immutable way all the main tender documents: that is, the information models presented both by the client and by the bidders.

The procedure described above guarantees the immutability and transparency of the tender documents published by the client. The procedures and criteria for evaluating the bids are accessible to all participants, making the reasons for the contract award explicit and eliminating any possible operational ambiguity. In the same way, the tenderers bids are also deposited in an unchangeable way on the distributed register, the client can access them only at the end of the reception phase, thus ensuring effective competition and giving all participants the opportunity to observe all the bids and to analyze them compared to the predefined award criteria.

\subsubsection{Construction}

The 4D and 5D dimensions model development allows to update and monitor the construction phase progress. The BIM model allows us to understand the actual construction works state, and the continuous information storage allows to understand the causes and trace those responsible in case of delays or budget excess. In this way, the client can control the actual activities progress and the sharing of information relating to the construction site is immediate and transparent.

The Blockchain used during the construction activity is also useful in controlling the supply chain: materials that arrive at the site are traced along the entire route and therefore in case of defects or delays you can consult each stage of processing that was recorded on the Blockchain database and then connected to it internally to the BIM model (Kouhizadeh and Sarkis 2018).

Finally, the record of both supplies and work status information allows the Smart Contract to be performed correctly. The connection between the information model, i.e., the activities progress, and the computational contract allows automatic payments to be issued whenever the milestone set by the work program is reached.

\subsubsection{Life Cycle}

Once the endeavor is completed, it is possible to create the as-built model of the building, containing all the information relating to each component actually built. Through the entire asset life cycle, the BIM model implementation based on Blockchain facilitates the satisfaction of the circular economy principles (Marzouk et al. 2018). Some 
building component data, collected during the construction phase and stored in the distributed registry, can support future maintenance, replacement, and dismission activities during the operational phase. The constant components conservation and maintenance state updating make it possible to use the BIM model as a database of materials and therefore to facilitate a considerable reduction in waste.

In addition, in the presence of system terminals equipped with smart interfaces, it is possible to envisage the execution of Smart Contract for maintenance work on plant devices in the building. Once the intervention has been carried out, in fact, the maintenance technician can insert the activity carried out into the machine, which confirms the fulfillment of Smart Contract clauses, automatically releasing the payment.

Considering this preliminary analysis of the integration of the BIM approach and Blockchain technology in the development of the procurement process, it is visible that one of the most encouraging advantages is the reduction of information asymmetry between all contracting parties. The data definition, updating, and validation by the participants allow them to access and consult, throughout the entire process, complete and truthful information, thus increasing mutual trust. Such a technological enhancement would ensure that operators have a transparent understanding of the procedures for the award of contracts, and that the client has a reliable check of the data relating to competitors, their offers and commitment.

\section{Conclusion}

The topics discussed in this paper demonstrate how the BIM model and Blockchain technology integration can offer benefits and add value to the main stages of contract execution. The BIM model can either incorporate information from Blockchain distributed ledger, or send to Blockchain information about model changes that need to be updated and used later in the execution of Smart Contract, for the automatic release of payments or the definition of new supply orders (Carson et al. 2018). The construction sector has always been characterized by aspects that, due to ambiguity or incompleteness, often generate conflicts during the contract execution. The potentials offered by the Blockchain and BIM model development allow to address positively the aforementioned problems thanks to applications that allow to streamline and improve the contract management, ensuring transparency in information sharing and traceability.

Despite the advantages, the main Blockchain obstacles are not only of a technical nature but involve other elements, including the man who decides to adopt the technology, economy, and institutions that must support and regulate the digital transition. In addition, because of the impossibility of predicting certain construction process variations, efficient Smart Contracts execution is rather difficult. 
For these reasons, unlike some industries, the construction sector has not yet developed an effective digital ecosystem and investments in new technologies. The integration between Blockchain technology and BIM is a key step in the sector progress: it creates a single source of true information about all aspects of the process, giving the digital project model the only reliable tool to support the development and management of all phases of the construction process-from the design phase to the operation along the whole lifecycle.

The Blockchain technology can, in fact, guide a lean construction process, by reducing the industry fragmentation and complexity, making it a single trusted entity. Blockchain technology is here and although it is at an early stage of development with many challenges it presents a relevant opportunity for all companies in the construction industry to emerge as more effective, transparent, and sustainable entities (Institution of Civil Engineers, Blockchain technology in the construction industry 2018).

\section{References}

Carson B, Romanelli G, Walsh P, Zhumaev A (2018) Blockchain beyond the hype: what is the strategic business value? McKinsey Co. pp 1-19

Di Giuda G, Villa V (2016) Il BIM. Guida completa al building information modeling per committenti, architetti, ingegneri, gestori immobiliari e imprese

Garzik J, Donnelly JC (2018) Blockchain 101: an introduction to the future. In: Handbook of Blockchain, Digital Finance and Inclusion, vol 2, pp 179-186. https://doi.org/10.1016/b978-012-812282-2.00008-5

Giancaspro M (2017) Is a 'smart contract' really a smart idea? Insights from a legal perspective. Comput Law Secur Rev. https://doi.org/10.1016/j.clsr.2017.05.007

Hsiao JI-H (2016) Smart contract on the blockchain—paradigm shift for contract law? https://doi. org/10.17265/1548-6605/2017.10.002

Institution of Civil Engineers, Blockchain technology in the construction industry (2018)

Kshetri N (2017) Blockchain's roles in strengthening cybersecurity and protecting privacy. Telecomm Policy 1027-1038. https://doi.org/10.1016/j.telpol.2017.09.003

Kshetri N (2018) Blockchain's roles in meeting key supply chain management objectives. Int J Inf Manag 39:80-89. https://doi.org/10.1016/j.ijinfomgt.2017.12.005

Kouhizadeh M, Sarkis J (2018) Blockchain practices, potentials, and perspectives in greening supply chains. https://doi.org/10.3390/su10103652

Marzouk M, Azab S, Metawie M (2018) BIM-based approach for optimizing life cycle costs of sustainable buildings. J Clean Prod 188:217-226. https://doi.org/10.1016/j.jclepro.2018.03.280

Mason J (2017) Intelligent contract and the construction industry. J Leg Aff Disput Resolut Eng Constr 9

Shen C, Pena-Mora F (2018) Blockchain for cities-a systematic literature review. IEEE Access 1-33. https://doi.org/10.1109/access.2018.2880744

Turk Ž, Klinc R (2017) Potentials of blockchain technology for construction management. Proc Eng 196:638-645. https://doi.org/10.1016/j.proeng.2017.08.052 
Open Access This chapter is licensed under the terms of the Creative Commons Attribution 4.0 International License (http://creativecommons.org/licenses/by/4.0/), which permits use, sharing, adaptation, distribution and reproduction in any medium or format, as long as you give appropriate credit to the original author(s) and the source, provide a link to the Creative Commons license and indicate if changes were made.

The images or other third party material in this chapter are included in the chapter's Creative Commons license, unless indicated otherwise in a credit line to the material. If material is not included in the chapter's Creative Commons license and your intended use is not permitted by statutory regulation or exceeds the permitted use, you will need to obtain permission directly from the copyright holder.

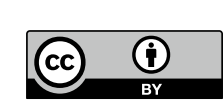

